\title{
Transcriptome Reveals That Genic SNPs Contributes to Heterosis in Cattle
}

\author{
Jiang-hong $W^{1,2, a, c}$, Husile GONG ${ }^{3, c}$, Qing-jiang $\operatorname{LUAN}^{1}$, Ling \\ XUE $^{4}$, Hai-lian SUN ${ }^{1,2}$ and Feng WANG ${ }^{1,2, b, *}$ \\ ${ }^{1}$ Animal husbandry Institute, Inner Mongolia Academy of Agricultural \& Animal \\ Husbandry Sciences, China; \\ ${ }^{2}$ Inner Mongolia Prataculture Research Center, Chinese Academy of Science, China; \\ ${ }^{3}$ College of Animal Science, Inner Mongolia Agricultural University, China; \\ ${ }^{4}$ Animal Husbandry Workstation of Duolun, China \\ awujianghonglong@126.com, ${ }^{\mathrm{b}} \mathrm{nmgwangfeng@163.com}$ \\ ${ }^{*}$ Corresponding author \\ ${ }^{\mathrm{C}} \mathrm{Co}$-first author
}

Keywords: Allele-specific gene expression, Coat color, Cattle, Transcriptome.

\begin{abstract}
Heterosis is a powerful and valuable tool for commercial beef producers, especially cow calf operations. Despite the importance of heterosis, and its extensive genetic analysis, little understanding exists of its molecular basis. We applied RNASeq technology to assess transcriptome-wide allele-specific gene expression (ASGE) in the hybrid genetic background at economic traits of offspring and maternal. 120 genes of these were different expression among three individuals in common. Among these genes, we found that three genes involved in melanogenesis pathway, including MC1R, DCT and TYR. In addition six of the altered expression genes were related to arachidonic acid (AA) metabolism, such as PTGS1, PTGIS, PTGR1, ALOX12B, ALOX15B and EPHX2. We find that coat color traits could be utilized to check the analysis pipeline for heterosis studies. Results from this study indicate that the parental alleles have un-equivalent functions in the hybrid, which may have an impact on heterosis.
\end{abstract}

\section{Introduction}

Genotype and phenotype mapping describes the basics of biological science[1]. Many phenotypic differences results from genetic variants and these are mainly mediated through changes in gene expression. Recently, developed next-generation high throughput RNA sequencing technology (RNA-Seq) has enabled the analysis of transcriptome-wide ASGE, facilitating the examination of allelic contributions to heterosis in hybrids [2]. Allelic expression bias in hybrids has been found to be correlated with parental differences, with trans-effects possibly mediating most hybrid transcriptional differences [3].

The impact of heterosis on different economic traits has been recognized for a long time, and structured crossbreeding programs have been designed in all livestock species to benefit from its effects [4]. Since hybrid gene expression is under the influence of two different and divergent genomes, gene expression levels in hybrids may reveal information about interactions of two parental alleles and their impacts [57]. Extensive gene expression level changes in hybrids relative to their parents have been documented in the past by a large number of studies (reviewed by [8]). These 
studies reported on extensive transcriptome remodelling in various tissues and developmental stages of various animal species heterotic hybrids[5]. It has also been hypothesized that differential gene expression in inbreds and hybrids contribute to heterosis[9]. Despite many reports regarding expression of specific genes within the cattle interspecies hybrid genetic background [10,11], thus far no attempt has been made to assess modulations in gene regulatory patterns on a global transcriptomic scale.

In this study, we focused our research on the black Hybrids F1 from a cross between black Wagyu and the maternal red Angus cattle. Red Angus is a red colored breed of beef cattle selected from the population of Aberdeen Angus cattle. Japanese black Wagyu is one of the most famous beef types within Japan and internationally, with a high fat-to-meat ratio. We applied RNA-Seq technology to assess transcriptome-wide ASGE in the hybrid genetic background at economic traits of offspring and maternal. Coat color is one of the most diverse phenotypic traits in livestock, and it exerts multiple adaptive functions, such as breeds identification, photoreception and thermoregulation. Coat color is a very useful trait for breeding because they can observe and relate to it easily. So to understand the molecular and biochemical mechanisms involved in heterosis, coat color traits could be utilized to check the analysis pipeline for heterosis studies. Results from this study indicate that the parental alleles have un-equivalent functions in the hybrid, which may have an impact on heterosis.

\section{Materials and Methods}

\section{Cattle Skin Sampling and Total RNA Extraction}

Three skin samples differing in their coat coloration (black coat and red coat) were collected from a three-way cross hybridization cattle family, which is result from the cross between one parent that is an F1 hybrid (Red Angus $\times$ Simmental) and Black Wagyu; include maternal red F1 hybrid and F2 hybrids twins(the Heifer and the Ox). Housing and care of cattle and collection of skin samples for use in the described experiments were conducted in accordance with the international council for laboratory animal science (http://iclas.org/wp-content/uploads/2012/07/ICLASEthical-Guidlines -Researcher.pdf).

A piece of skin $(1 \mathrm{~cm}$ in diameter) from the neck was collected via punch skin biopsy under local anesthesia and immediately placed in liquid nitrogen. Total RNA from the sample was extracted using Trizol reagent (Invitrogen, USA) according to the manufacturer's instructions. The RNA integrity was evaluated by gel electrophoresis and the RNA purity was checked by the ratio of OD260/OD280 and RIN value. RNA samples with RIN value greater than 8.4 and OD260/OD280 ratio greater than 1.7 were selected for deep sequencing.

\section{Preparation and Sequencing (mRNA-seq) of the cDNA Library}

The workflow described in this section is shown in Figure S1. In brief, RNA was collected from skin tissues, and oligo(dT) beads were used to isolate poly(A) mRNA. Fragmentation buffer was used to chop the mRNA into short fragments, which were then used as templates for random hexamer-primed synthesis of first-strand cDNA. Second-strand cDNA was synthesized using buffer, dNTPs, RNase H, and DNA polymerase I. From these cDNA, a paired-end library was synthesized using the Genomic Sample Prep kit (Illumina), according to the manufacturer's instructions. Short fragments were purified with the QIAquick PCR (Qiagen) extraction kit and 
then resolved with EB buffer for end repair and the addition of poly (A). The short fragments were then connected with sequencing adapters, and suitable fragments were separated by agarose gel electrophoresis. Finally, the sequencing library was built by PCR amplification and sequenced using the HiSeq ${ }^{\text {TM }} 2500$ platform (Illumina).

\section{Reads Alignment to the Bovine Reference Genome and SNP Calling}

The Cattle reference genome (UMD3.1) and corresponding gene model annotation files were downloaded directly from the cow genome website (ftp://ftp.ensembl.org/pub/release-79/fasta/ bos_taurus/dna/). An index of the reference genome was built using Bowtie v0.12.8[12] and paired-end clean reads for each individual were aligned to the reference genome by TopHat v2.0.0 (http://tophat.cbcb.umd.edu/)[13]. Only reliable properly paired BWA mapped reads were considered for Single Nucleotide Polymorphism (SNP) calling. SNPs were called using the SAMtools software package[14]. Genotype likelihoods were computed using the SAMtools utilities and variable positions in the aligned reads compared to the reference were called with the BCFtools utilities.

\section{Identification of Differentially Expressed Genes}

The numbers of RNA-seq reads produced from a transcript is directly proportional to its abundance; therefore, the gene expression level could be quantified by the reads count. RSEM v1.1117 with default parameters on these alignments to estimate expression levels, and then the RSEM-estimated fragment counts were fed into DESeq package (1.0.6) to get the 'baseMean' value. The false discovery rate (FDR) of each comparison (mother vs. son and mother vs. daughter) was calculated by the winflat program which implements a rigorous statistical analysis described by Audic and Claverie. The FDR $\leq 0.01$ and the absolute value of $\log 2$ ratio $\geq 1$ were used as the threshold of significant differences in gene expression ${ }^{[15]}$. Those genes that were significantly differentially expressed in both mother vs. son and mother vs. daughter were identified as potentially related to skin color and other phenotypes.

\section{Selection of Candidate SNPs from Transcriptomes}

Because that the offspring of twins resulting from artificial insemination, the father skin sample has not been obtained. If F1 hybrid has two different alleles (code variants) at a specific gene locus, then the individual is heterozygous at that locus. While mater has two same alleles at that locus, other copy allele must be from male parent. In order to investigate how the alleles of parents contribute to heterosis of progeny, we selected the intersection of homozygosis SNPs of mater and heterozygosis SNPs of progeny for subsequent analysis. Heterozygous SNPs were identified from genomic DNA using TopHat2.0, and the allelic counts at these sites were extracted from the bam files using Samtools[14].

\section{Hypergeometric Testing on Annotations and Gene Ontology (GO) Visualization}

Hypergeometric tests were performed on annotations using updated databases from The Gene Ontology for GO categories from Biological Process and Kyoto Encyclopedia of Genes and Genomes for pathways in ClueGO[16]. Only genes with FDR corrected $\mathrm{p}(\mathrm{q}) \leq 0.05$ and a fold-regulation $>2$ in a pairwise compartment comparison were included in the hypergeometric testing. The ClueGO output was restricted to GO categories with $\mathrm{q} \leq 0.05$, and the GO term fusion was used to avoid redundancy prior to visualization of networks in the Cytoscape environment [17]. 


\section{Results}

\section{Deep Sequencing and Mapping of RNA-seq Reads}

RNA-seq technology has proven to be an efficient way to uncover structures of transcriptomes and to assess allele-specific gene expression (Pastinen, 2010). To measure ASGE patterns in a bovine interspecies model, RNA-seq was employed to amplify and sequence cDNA fragments from a hybridation family: F1 hybrid (Red Angus $\times$ Simmental) and their F2 interspecies hybrids. These sequencing runs produced from 41 to 50 million $150 \mathrm{bp}$ paired ends reads for these samples (Table 1). RNA-seq reads were mapped to a reference transcriptome de novo assembled from 3 samples together. This reference transcriptome contains 285,001 transcripts with an average length of $559 \mathrm{bp}$ (Table 2).

Table1. Overview of Mapping Status

\begin{tabular}{|c|c|c|c|c|c|}
\hline samples & $\begin{array}{r}\text { Total } \\
\text { Reads }\end{array}$ & $\begin{array}{r}\text { Mapped } \\
\text { Reads } \\
\end{array}$ & $\begin{array}{r}\text { Mapped } \\
\text { Ratio } \\
\end{array}$ & $\begin{array}{ll}\text { Uniq } & \text { Mapped } \\
\text { Reads } & \\
\end{array}$ & $\begin{array}{r}\text { Uniq Mapped } \\
\text { Ratio }\end{array}$ \\
\hline Mother & $\begin{array}{r}45,538,21 \\
6\end{array}$ & $38,042,905$ & $83.54 \%$ & $36,795,162$ & $80.80 \%$ \\
\hline Son & $\begin{array}{r}50,397,55 \\
4\end{array}$ & $42,413,824$ & $84.16 \%$ & $41,341,885$ & $82.03 \%$ \\
\hline Daughter & $\begin{array}{r}41,594,85 \\
6\end{array}$ & $35,122,916$ & $84.44 \%$ & $34,325,424$ & $82.52 \%$ \\
\hline
\end{tabular}

Table2. Assembly statistics for three cattle transcriptomes

\begin{tabular}{lllll}
\hline Parameter & Maternal & Son & Daughter & Together \\
\hline No. of reads & 45538216 & 50397554 & 41594856 & 137530626 \\
No. of transcripts & 210282 & 210754 & 236258 & 285001 \\
No. of Trinity components & 149057 & 144480 & 147491 & 167340 \\
Transcriptome size & $256 \mathrm{M}$ & $277 \mathrm{M}$ & $384 \mathrm{M}$ & $485 \mathrm{M}$ \\
Average length & 401 & 434 & 532 & 559 \\
Median length & 1155.30 & 1250.77 & 1551.10 & 1619.11 \\
\hline
\end{tabular}

\section{Identification SNPs in Cow Hybridizing Family}

For SNP calling, BWA was used to map the paired-reads from each sample to the bovine reference genome sequence. The SAM tools package was used for SNP discovery using stringent parameters (e.g. minimum coverage of 8 reads and mapping quality of 20). SAMtools can identify single base substitutions as well as small insertions and deletions; however, only SNPs were considered in the current analysis. In total 141,699 different SNP positions were detected with the RNA-Seq reads. Amongst these SNPs, almost 65\% were heterozygous in all three sequenced samples, corresponding presumably to differences between F1 hybrid and their F2 interspecies hybrids (Table3). 


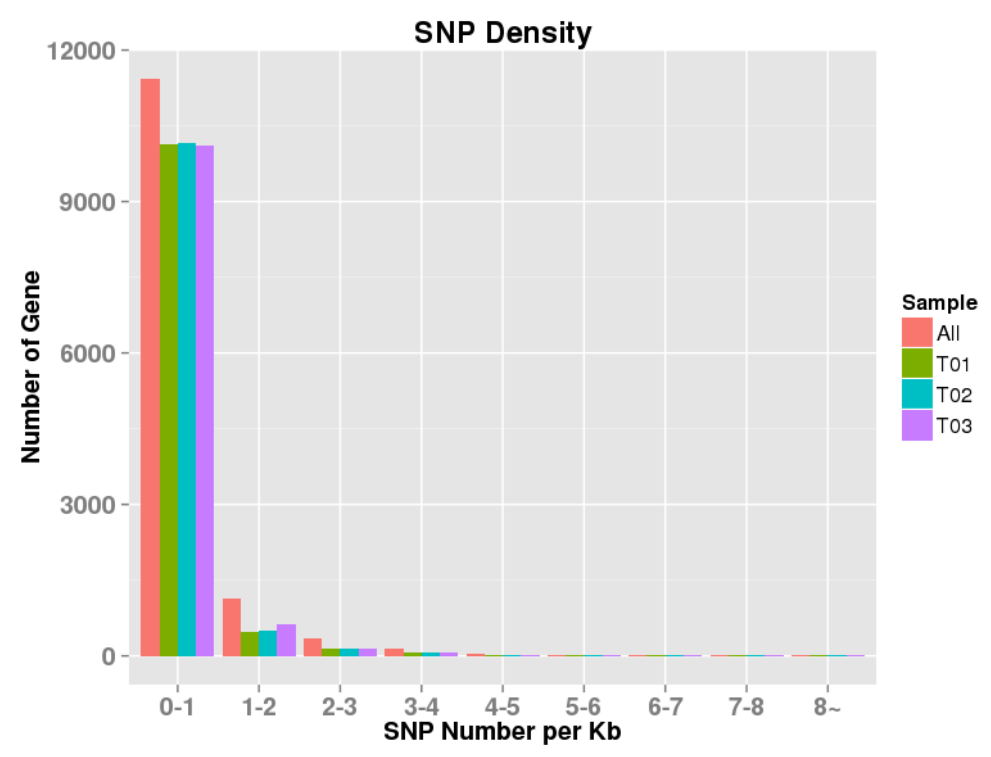

Figure1. The density distribution of SNPs in three cattle transcriptomes, T01, mother, T02, son, T03, daughter.

Table3. SNPs statistics for three cattle transcriptomes

\begin{tabular}{ccccccc}
\hline $\begin{array}{c}\text { Sample } \\
\text { ID }\end{array}$ & $\begin{array}{c}\text { SNP } \\
\text { Number }\end{array}$ & $\begin{array}{c}\text { Genic } \\
\text { SNP }\end{array}$ & $\begin{array}{c}\text { Intergenic } \\
\text { SNP }\end{array}$ & $\begin{array}{c}\text { Transiti } \\
\text { on }\end{array}$ & $\begin{array}{c}\text { Transvers } \\
\text { ion }\end{array}$ & $\begin{array}{c}\text { Heterozygo } \\
\text { sity }\end{array}$ \\
\hline Mother & 71,106 & 45370 & 26587 & $71.52 \%$ & $28.48 \%$ & $65.45 \%$ \\
Son & 70,350 & 45732 & 25522 & $72.22 \%$ & $27.78 \%$ & $65.86 \%$ \\
Daughter & 73,859 & 49928 & 24870 & $72.82 \%$ & $27.18 \%$ & $65.85 \%$ \\
\hline
\end{tabular}

In our study, firstly, homozygosis SNPs of mater and heterozygosis SNPs of progeny were extracted. Then, we take the intersection of two subset SNPs of mother and son, respectively (Figure1). These SNP sets of two were considered in the subsequent analysis. We found that there were 67,887 homozygosity SNPs mapping to coding regions in mother. The set, these SNPs intersects with heterozygosity SNPs of progeny, which contains 13,031 SNPs. These SNPs were distributed on 4905 genes.

\section{Differential Gene Expression between Mater and Progeny}

We have analyzed the expression levels of those genes above in three individuals. In total, 488 genes were detected as significantly different by EBSeq (Fold Change $\geq 2$, FDR $<0.01)$. Of the 488 genes, 214 were different expressing between mother and male progeny, and 394 were different expressing between dam and female offspring. 120 genes of these were different expression among three individuals in common(Figure2B-C).

(a) Hierarchical clustering of transcripts and samples. Shown is a heat map showing the relative expression levels of each transcript (rows) in each sample (column). Rows and columns are hierarchically clustered. Expression values (FPKM) are $\log 2$ transformed and then median-centered by transcript.

(b) Volcano plot reporting false discovery rate $(-\log 10 \mathrm{FDR}, \mathrm{y}$ axis) as a function of $\log 2$ (fold change) between mother and son ( $\log F C, x$ axis). Transcripts that are identified as significantly differentially expressed at most $0.1 \%$ FDR are colored in red.

(c) Volcano plot reporting false discovery rate $(-\log 10 \mathrm{FDR}, \mathrm{y}$ axis) as a function of $\log 2$ (fold change) between mother and daughter $(\operatorname{logFC}, \mathrm{x}$ axis). Transcripts that are 
identified as significantly differentially expressed at most $0.1 \%$ FDR are colored in red.

(d) Venn diagram of different expressed genes.

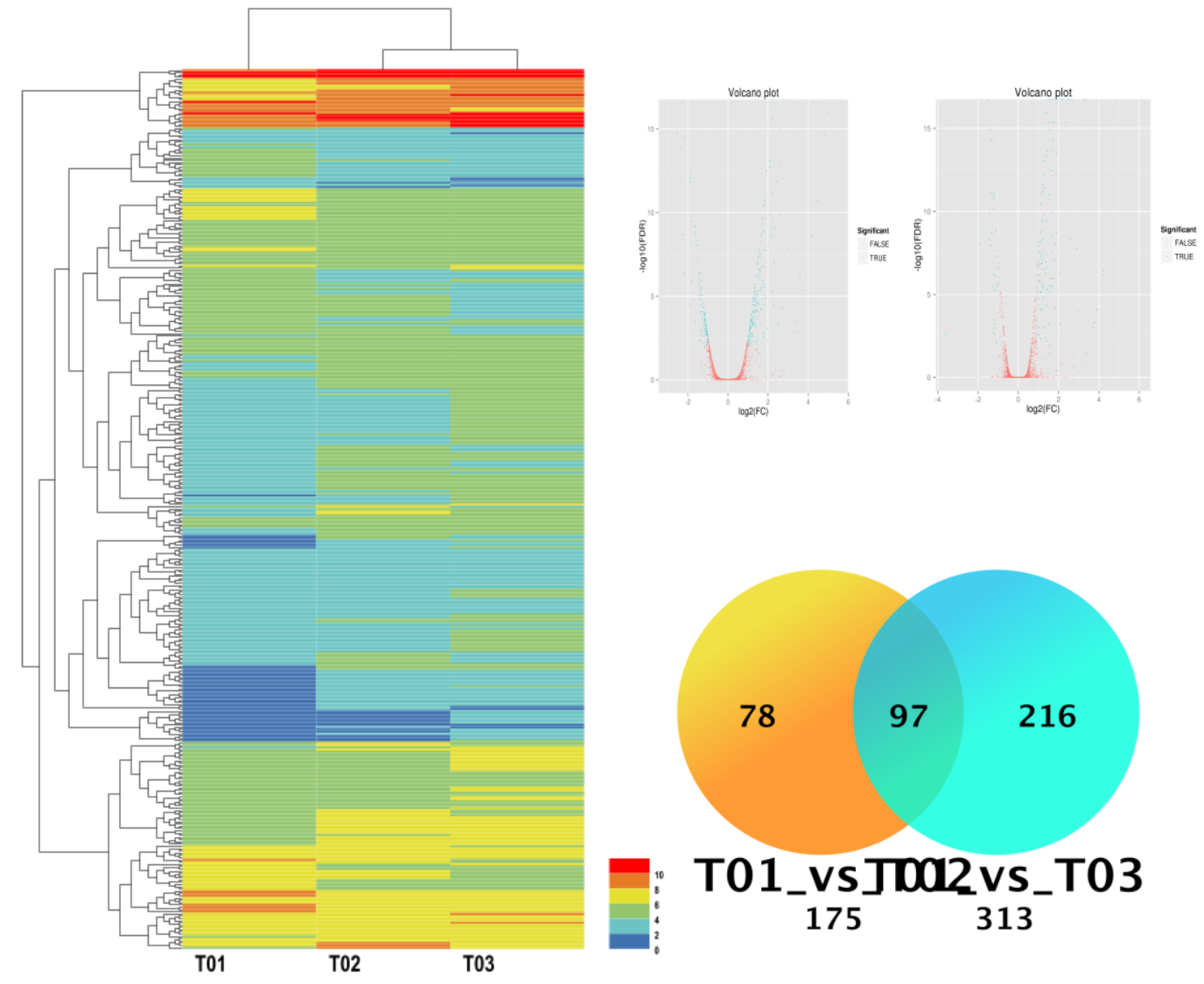

Figure2. Comparisons of transcriptional profiles across samples. T01, Mother, T02, Son, T03, Daughter.

\section{Function Analysis of Different Expression Genes}

In order to analyze the pathway of metabolism and gene ontology for different expression genes (DEGs), we utilized the ClueGO to integrate the Gene Ontology (GO) terms and KEGG pathway terms and create a functionally organised GO/KEGG network. Functional annotation with biological processes category resulted in 260 (66.49\%) genes found from cluster for 391 DEGs (Figure2D), while KEGG pathway category resulted in $126(32.23 \%)$ genes found from cluster.

The ClueGO overview pie chart highlighted that significant proportion of the genes differentially regulated are involved in "melanin biosynthetic and metabolism" and "fat acids metabolism" by both GO (biological process) and KEGG pathway. Majority of the genes are involved in "immune response", as shown by both KEGG and GO (figure 3). 


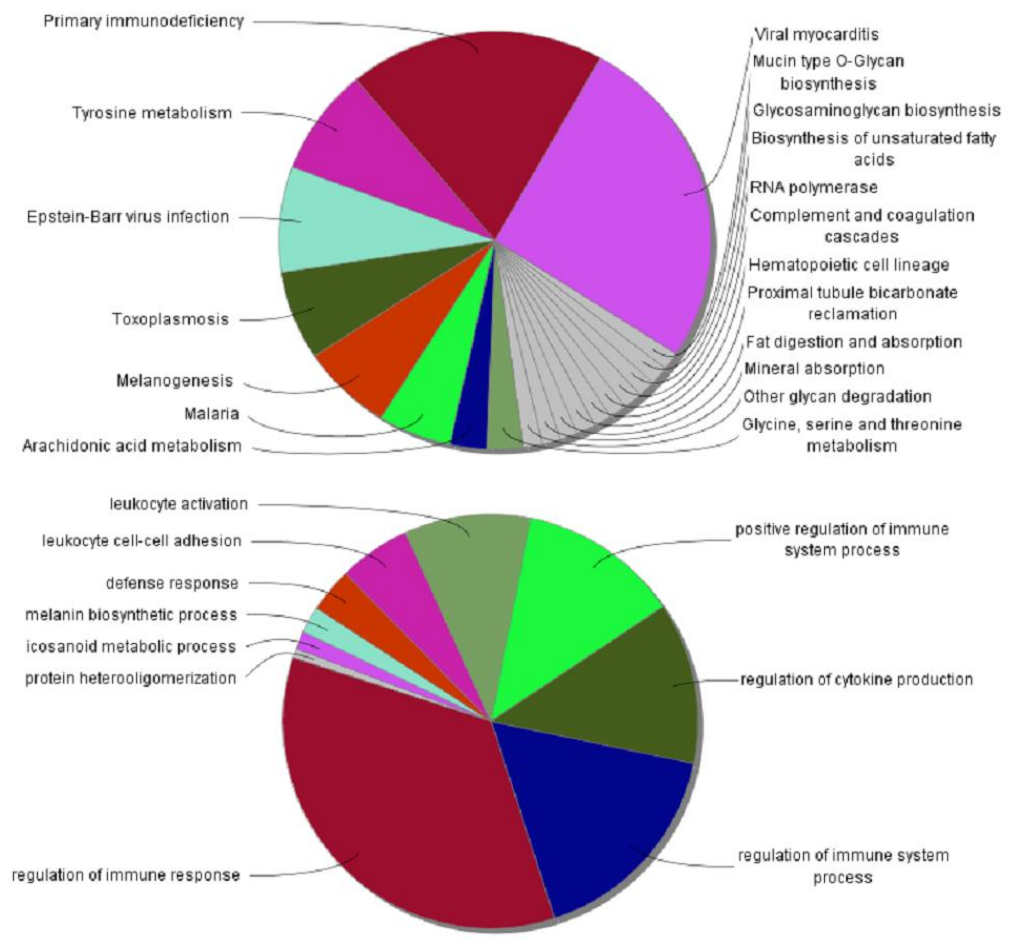

Figure3. Function annotations of different expression genes, A, GO-biological process; B, KEGG.

\section{KEGG Pathway Analysis}

Of the 488 genes differentially expressed in progeny versus mater skin, 126 had a specific KEGG pathway annotation. 17 of these genes were associated with three pathways including those functionally related to coat color in skin such as Melanogenesis, Mineral absorption, Tyrosine metabolism. Remaining KEGG pathway annotated genes were associated with three pathways including those functionally related to Fat acid metabolism such as Arachidonic acid metabolism, Biosynthesis of unsaturated fatty acids and Fat digestion and absorption. The enriched GO terms for genes identified in skin transcriptome related to pigmentation and melanogenesis and their relative expression in black versus red skin are shown in Table 4 and Figure 4.

Table4. The information of interesting cluster and genes

\begin{tabular}{|c|c|c|}
\hline Cluster & erms & bols \\
\hline Pigment & $\begin{array}{l}\text { Aelanogenesis(KEGG) } \\
\text { Gineral absorption(KEGG) } \\
\text { yrosine metabolism(KEGG) } \\
\text { Melanogenesis (GO) } \\
\text { Gineral absorption(GO) }\end{array}$ & 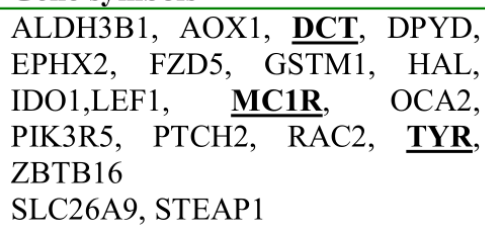 \\
\hline $\begin{array}{l}\text { Fat acid } \\
\text { metabolism } \\
\text { associated }\end{array}$ & $\begin{array}{l}\text { Arachidonic acid metabolism (KEGG) } \\
\text { Biosynthesis of unsaturated fatty acids(KEGG) } \\
\text { Fat digestion and absorption(KEGG) } \\
\text { icosanoid metabolic process (GO) } \\
\text { fatty acid derivative metabolic process }(\mathrm{GO})\end{array}$ & $\begin{array}{l}\frac{\text { ALOX12B }}{\text { CD36, }}, \underline{\text { ALOX15B }}, \frac{\text { ALOX5 }}{\text { DAGLA, }}, \\
\text { HSD17B12, } \quad \text { LOC100847677, } \\
\text { MOGAT2, MGLL, PLA2G2F, } \\
\text { PTGIS, PTGS1, PECR, PTGR1 }\end{array}$ \\
\hline
\end{tabular}

NOTE: those genes were found by GO and KEGG in common, which shown in boldface and underline. 


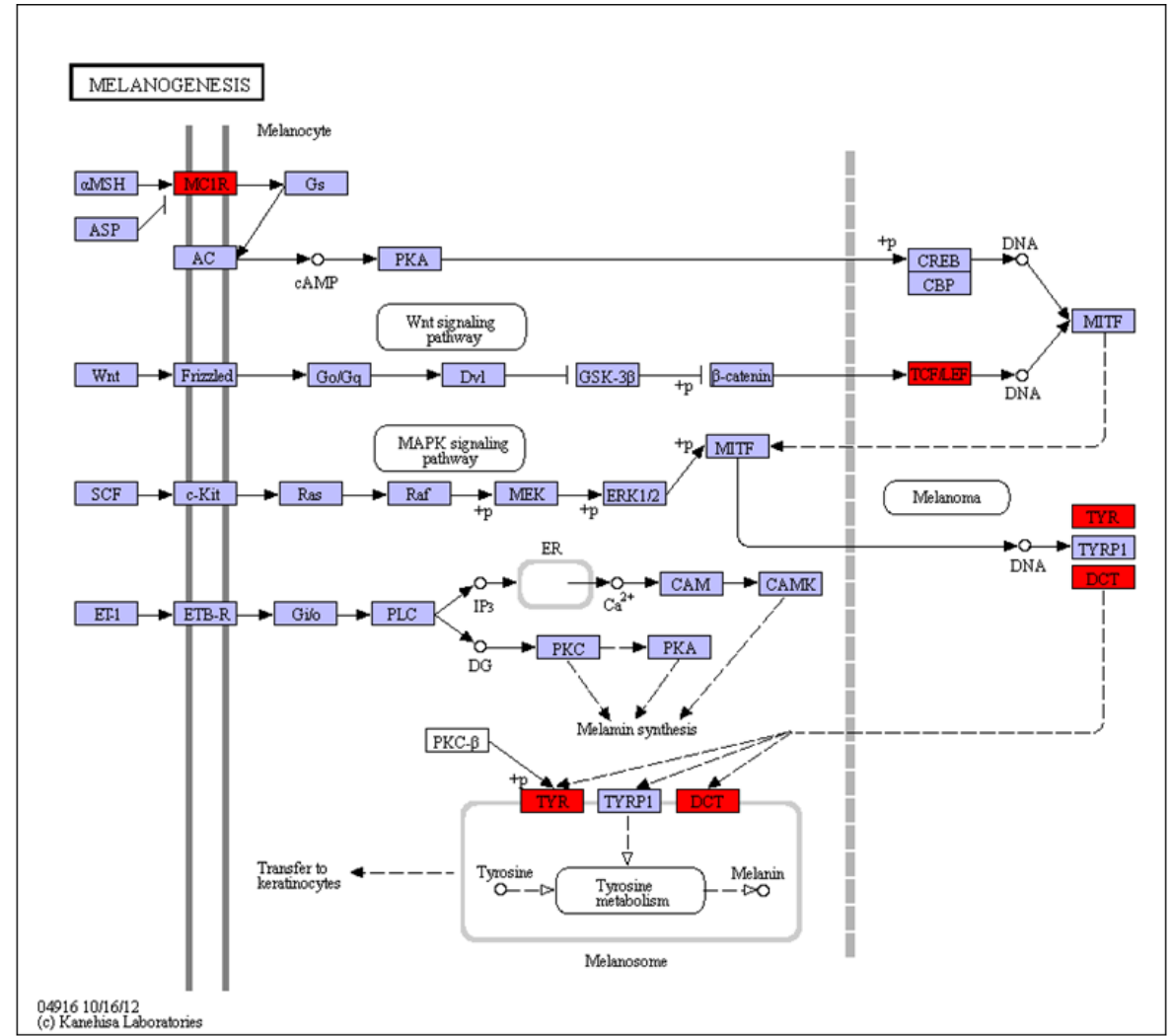

Figure4. Differentially expressed coat color genes with SNPs in cattle hybrids family and their involvement in the melanogenesis pathway.

\section{SNP for Interesting Genes}

To further investigate allele specific expression bias of interesting genes in the F1 hybrids, 34 genes in table were analyzed. For each SNP site, calculate the number of REF and ALT read overlaps in these genes (Table4). For MC1R, the paternally inherited allele of exonic SNP 296C was predominantly expressed over the allele of SNP 296T in two offspring individuals. This change alters the encoded amino acid sequence in coding region, which results in the leu99pro amino acid change. DCT gene has two SNPs (3'UTR.21C>A, 3'UTR.296C>T, 3'UTR.570T>C).

A total of twelve SNPs in PTGS1 of F1 hybrids including four nonsynonymous SNPs (c.194A $>$ C, c.394G $>A$, c.835A $>$ G, c.898G $>$ A) that introduce amino acid changes and five synonymous SNPs (c.57A $>\mathrm{G}$, c.780G $>\mathrm{A}$, c.972C $>\mathrm{T}$, c.1251G $>\mathrm{T}$ and c. 1365G $>$ T) in coding sequence and three SNPs (3'UTR.21C $>$ A, 3'UTR.296C $>$ T, 3'UTR.570T $>$ C) in 3'UTR were detected, revealing high genetic diversity(Figure5). However, these sites are almost homozygotic in mother. These results show that hybridization can increase genetic diversity. 
Table5. The number of allelic base read overlaps of interesting genes

\begin{tabular}{|c|c|c|c|c|}
\hline & Allelic base & Mother & S2-1 & $\mathrm{S} 2-2$ \\
\hline \multirow{2}{*}{ MC1R-pos 296} & $\mathrm{~T}$ & 3 & 21 & 7 \\
\hline & $\mathrm{C}$ & 0 & 39 & 14 \\
\hline \multirow{2}{*}{ ALOX12B-pos 949} & G & 0 & 8 & 19 \\
\hline & $\mathrm{A}$ & 3 & 4 & 14 \\
\hline \multirow{2}{*}{ ALOX15B-pos 949} & A & 127 & 50 & 23 \\
\hline & G & 0 & 13 & 16 \\
\hline \multirow{2}{*}{ PTGS1, c.57A $>$ G } & $\mathrm{A}$ & 0 & 29 & 34 \\
\hline & $\mathrm{G}$ & 41 & 36 & 60 \\
\hline \multirow{2}{*}{ PTGS1, c.194A >C } & $\mathrm{A}$ & 31 & 25 & 50 \\
\hline & $\mathrm{C}$ & 0 & 10 & 42 \\
\hline \multirow{2}{*}{ PTGS1, c.394G $>$ A } & G & 44 & 31 & 54 \\
\hline & A & 0 & 24 & 47 \\
\hline \multirow{2}{*}{ PTGS1, c.780G $>$ A } & G & 41 & 37 & 70 \\
\hline & $\mathrm{A}$ & 0 & 33 & 32 \\
\hline \multirow{2}{*}{ PTGS1, c.835A $>\mathrm{G}$} & $\mathrm{A}$ & 41 & 40 & 76 \\
\hline & G & 0 & 30 & 40 \\
\hline \multirow{2}{*}{ PTGS1, c. $898 \mathrm{G}>\mathrm{C}$} & G & 43 & 53 & 93 \\
\hline & $\mathrm{C}$ & 0 & 27 & 48 \\
\hline \multirow{2}{*}{ PTGS1, c. $972 \mathrm{C}>\mathrm{T}$} & $\mathrm{C}$ & 41 & 43 & 71 \\
\hline & $\mathrm{T}$ & 0 & 22 & 37 \\
\hline \multirow{2}{*}{ PTGS1, c. $1251 \mathrm{C}>\mathrm{T}$} & $\mathrm{C}$ & 52 & 17 & 79 \\
\hline & $\mathrm{T}$ & 0 & 15 & 28 \\
\hline \multirow{2}{*}{ PTGS1, c. $1365 \mathrm{G}>\mathrm{T}$} & $\mathrm{G}$ & 66 & 51 & 90 \\
\hline & $\mathrm{T}$ & 0 & 38 & 62 \\
\hline \multirow{2}{*}{ PTGS1, 3'utr 21} & $\mathrm{C}$ & 56 & 38 & 50 \\
\hline & A & 0 & 28 & 33 \\
\hline \multirow{2}{*}{ PTGS1, 3'utr 296} & $\mathrm{C}$ & 49 & 42 & 75 \\
\hline & $\mathrm{T}$ & 0 & 31 & 33 \\
\hline \multirow{2}{*}{ PTGS1, 3'utr 570} & $\mathrm{~T}$ & 41 & 18 & 33 \\
\hline & $\mathrm{C}$ & 0 & 21 & 31 \\
\hline
\end{tabular}

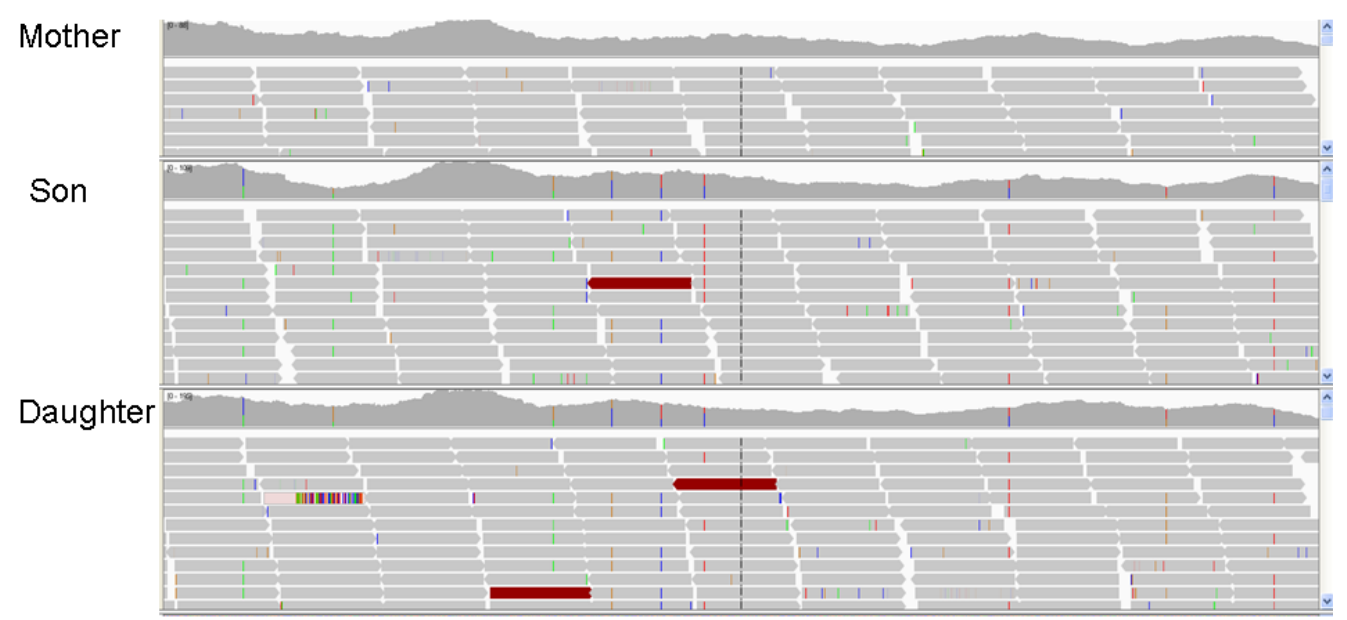

Figure5. The location of SNPs in PTGS1 gene of cattle hybrids family (IGV view)

\section{Discussion}

The advantages of crossbreeding in beef cattle production systems are widely recognized (Long, 1980), one of the major reasons being the benefit arising from heterosis, which may result in improved quality of beef, such as flavour and leanness. 
In this study, we used a transcriptome sequencing approach to examine the allele specific expression of two hybrids families for heterosis of progeny. Coat color and pattern have been a hallmark characteristic of many breeds of cattle since registries began, and therefore, undoubtedly before. Coat color is a very useful trait for breeding because they can observe and relate to it easily. So we utilize coat color traits to check the analysis pipeline for heterosis studies. Four candidate genes of melanogenesis pathway, including MC1R, DCT, TYR and LEF1 genes, were enriched within KEGG pathway. MC1R, one of these genes, was predominantly expressed over the paternally inherited allele of SNP 296T in two offspring individuals. This change alters (c.296C $>\mathrm{T}$ ) the encoded amino acid sequence in coding region, which results in the leu99pro amino acid change and dominant allele $E^{\mathrm{D}}$ appear $\left(E^{\mathrm{D}}>E^{+}\right)$. Each offspring carry a maternal red Angus allele $\left(E^{+}\right)$and a paternal Black Wagyu allele $\left(E^{\mathrm{D}}\right)$ at MC1R gene locus. In cattle, black and red coat color are postulated to be produced by E- and A-locus alleles[18]. These two alleles were postulated at the Elocus from color inheritance studies; $E^{\mathrm{D}}$ for dominant black and the $E^{+}$allele, which allows phenotypical expression of A-locus alleles [19]. The dominant $E^{\mathrm{D}}$ allele produces a constitutively active MSH-receptor that is insensitive to any A-locus product. This results in an increased tyrosinase level within the melanocytes and eumelanin synthesis [20]. Therefore, our analysis pipeline is doable to research the heterosis of progeny, especially for qualitative trait.

And then, we found some candidate genes associated with fat acid metabolism associated by this pipeline. Six genes of Arachidonic acid metabolism were enriched within KEGG pathway. Our candidate genes included PTGS1 and PTGIS, two central enzymes in the biosynthetic pathway of prostaglandins from AA; ALOX5[21], ALOX12B, and ALOX15B, which catalyze the conversion of fat acids hydroperoxides from Polyunsaturated fatty acids; and EPHX2, which plays a major role in the in vivo metabolism of endogenous lipid epoxides, such as the epoxyeicosatrienoic acids and squalene oxide, a key intermediate in the synthesis of cholesterol[22]. We investigated both candidate polymorphisms with defined functional impact and SNPs. These results indicate that the AA metabolism pathway may be involved in the beef quality of hybrid. AA is an $n-6$ polyunsaturated fatty acid present in animal lipids. Recent studies have shown that the palatability of chicken meat can be improved by dietary arachidonic acid supplementation[23]. We find twelve SNPs in PTGS1, revealing high genetic diversity of F1 hybrids.

Allelic variation of gene expression is common in cattle, and is of interest because of its potential contribution to variation in heritable traits. Transcriptional activity at the different alleles of a gene in a diploid genome can differ considerably. Both genetic and epigenetic determinants govern this ASGE and this highly regulated process can lead to diversity of progeny. Allele-specific expression refers to the preferential expression of one of the two alleles in a diploid genome, which has been thought largely attributable to the associated cis-element variation and allele-specific epigenetic modification patterns. Allele-specific expression may contribute to the heterosis (or hybrid vigor) effect in hybrid livestock that are produced from crosses of closely-related species, subspecies and/or inbred lines.

\section{Conclusions}

In our study, transcriptome sequencing was employed to identify allele gene-specific expression in hybrid family. 120 genes of these were different expression among three individuals in common. Among these genes, we found that six of the altered 
expression genes were related to arachidonic acid (AA) metabolism, such as PTGS1, PTGIS, PTGR1, ALOX12B, ALOX15B and EPHX2. In addition four genes involved in melanogenesis pathway, including MC1R, DCT and TYR. Results from this study indicate that the parental alleles have un-equivalent functions in the hybrid, which may have an impact on heterosis. The use of ASGE for association transcriptomics approaches such as ours requires further development and evaluation before it might be a suitable adjunct to breeding programs.

\section{Authors' Contributions}

JHW and FW conceived the study; JHW performed analysis of RNA-seq data, and drafted the manuscript. QJL, LX and HLS prepared the sample. JHW and HG performed the data analysis. All authors critically read the manuscript drafts and approved the final version of the manuscript.

\section{Acknowledgements}

This work was supported by Projects in the National Science \& Technology Pillar Program (Grant No. 2012BAD13B02) and Innovation Foundation of IMAAAHS (Grant No. 2016CXJJM10, 2017CXJJM03-2), also National Natural Science Foundation of China (Grant No. 31560623).

\section{References}

[1] Rockman M V Reverse engineering the genotype-phenotype map with natural genetic variation [J]. Nature. 2008, 456(7223): 738-44.

[2] Skelly D A, M Johansson, J Madeoy, et al. A powerful and flexible statistical framework for testing hypotheses of allele-specific gene expression from RNA-seq data [J]. Genome Res. 2011, 21(10): 1728-37.

[3] He G, X Zhu, A A Elling, et al. Global epigenetic and transcriptional trends among two rice subspecies and their reciprocal hybrids[J]. Plant Cell. 2010, 22(1): 1733.

[4] Gama L T, M C Bressan, E C Rodrigues, et al. Heterosis for meat quality and fatty acid profiles in crosses among Bos indicus and Bos taurus finished on pasture or grain [J]. Meat Sci. 2013, 93(1): 98-104.

[5] Shen Y, J Catchen, T Garcia, et al. Identification of transcriptome SNPs between Xiphophorus lines and species for assessing allele specific gene expression within F-1 interspecies hybrids[J]. Comparative Biochemistry and Physiology C-Toxicology \& Pharmacology. 2012, 155(1): 102-108.

[6] Hegarty M J, G L Barker, A C Brennan, et al. Extreme changes to gene expression associated with homoploid hybrid speciation [J]. Mol Ecol. 2009, 18(5): 877-89.

[7] Wolf J B, R J Oakey,R Feil Imprinted gene expression in hybrids: perturbed mechanisms and evolutionary implications[J]. Heredity (Edinb). 2014, 113(2): 167-75.

[8] Hochholdinger F, N Hoecker Towards the molecular basis of heterosis[J]. Trends Plant Sci. 2007, 12(9): 427-32. 
[9] Stupar R M, J M Gardiner, A G Oldre, et al. Gene expression analyses in maize inbreds and hybrids with varying levels of heterosis[J]. BMC Plant Biol. 2008, 833.

[10] Ahlberg C M, L N Schiermiester, J T, et al. Genome wide association study of cholesterol and poly- and monounsaturated fatty acids, protein, and mineral content of beef from crossbred cattle[J]. Meat Sci. 2014, 98(4): 804-14.

[11]Liu Z, Q Li, Q Zhang, et al. Analysis of IGF2 mRNA expression and its methylation status between cattle yaks and their parents[J]. Progress in Natural Science. 2009, 19(9): 1063-1069.

[12] Giannoulatou E, S H Park, D T Humphreys, et al. Verification and validation of bioinformatics software without a gold standard: a case study of BWA and Bowtie[J]. BMC Bioinformatics. 2014, 15 Suppl 16S15.

[13] Trapnell C, A Roberts, L Goff, et al. Differential gene and transcript expression analysis of RNA-seq experiments with TopHat and Cufflinks[J]. Nat Protoc. 2012, 7(3): 562-78.

[14]Li H, B Handsaker, A Wysoker, et al. The Sequence Alignment/Map format and SAMtools[J]. Bioinformatics. 2009, 25(16): 2078-9.

[15]Love M I, W Huber,S Anders Moderated estimation of fold change and dispersion for RNA-seq data with DESeq2[J]. Genome Biol. 2014, 15(12): 550.

[16] Bindea G, B Mlecnik, H Hackl, et al. ClueGO: a Cytoscape plug-in to decipher functionally grouped gene ontology and pathway annotation networks[J]. Bioinformatics. 2009, 25(8): 1091-3.

[17] Shannon P, A Markiel, O Ozier, et al. Cytoscape: a software environment for integrated models of biomolecular interaction networks[J]. Genome research. 2003, 13(11): 2498-2504.

[18] Adalsteinsson S, S Bjarnadottir, D I Vage, et al. Brown coat color in Icelandic cattle produced by the loci Extension and Agouti [J]. J Hered. 1995, 86(5): 395-8.

[19] Klungland H, D I Vage, L Gomez-Raya, et al. The role of melanocyte-stimulating hormone (MSH) receptor in bovine coat color determination [J]. Mamm Genome. 1995, 6(9): 636-9.

[20] Robbins L S, J H Nadeau, K R Johnson, et al. Pigmentation phenotypes of variant extension locus alleles result from point mutations that alter MSH receptor function[J]. Cell. 1993, 72(6): 827-34.

[21] Powell W S, J Rokach The eosinophil chemoattractant 5-oxo-ETE and the OXE receptor [J]. Prog Lipid Res. 2013, 52(4): 651-65.

[22] Morisseau C, B D Hammock Impact of soluble epoxide hydrolase and epoxyeicosanoids on human health [J]. Annu Rev Pharmacol Toxicol. 2013, 5337-58.

[23] Kiyohara R, S Yamaguchi, K Rikimaru, et al. Supplemental arachidonic acidenriched oil improves the taste of thigh meat of Hinai-jidori chickens[J]. Poult Sci. 2011, 90(8): 1817-22. 